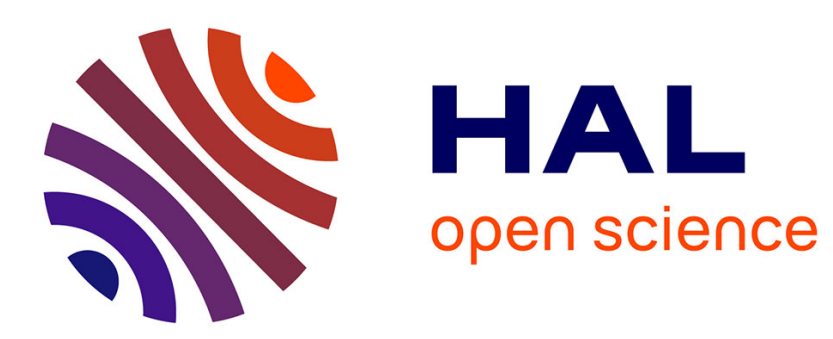

\title{
Task-Oriented Conversational Behavior of Agents for Collaboration in Human-Agent Teamwork
}

Mukesh Barange, Alexandre Kabil, Camille de Keukelaere, Pierre Chevaillier

\section{To cite this version:}

Mukesh Barange, Alexandre Kabil, Camille de Keukelaere, Pierre Chevaillier. Task-Oriented Conversational Behavior of Agents for Collaboration in Human-Agent Teamwork. PAAMS, Jun 2014, Salamanca, Spain. pp.25-37, 10.1007/978-3-319-07551-8_3 . hal-01165931

\section{HAL Id: hal-01165931 \\ https://hal.science/hal-01165931}

Submitted on 22 Jun 2015

HAL is a multi-disciplinary open access archive for the deposit and dissemination of scientific research documents, whether they are published or not. The documents may come from teaching and research institutions in France or abroad, or from public or private research centers.
L'archive ouverte pluridisciplinaire HAL, est destinée au dépôt et à la diffusion de documents scientifiques de niveau recherche, publiés ou non, émanant des établissements d'enseignement et de recherche français ou étrangers, des laboratoires publics ou privés.

\section{()ㅜ) $\Theta$}

Distributed under a Creative Commons Attribution - NoDerivatives| 4.0 International 


\title{
Task-Oriented Conversational Behavior of Agents for Collaboration in Human-Agent Teamwork
}

\author{
Mukesh Barange, Alexandre Kabil, Camille De Keukelaere, and Pierre \\ Chevaillier \\ ENIB-UEB; Lab-STICC, France
}

\begin{abstract}
Coordination is an essential ingredient for human-agent teamwork. It requires team members to share knowledge to establish common grounding and mutual awareness among them. This paper proposes a behavioral architecture $C^{2} B D I$ that enhances the knowledge sharing using natural language communication between team members. Collaborative conversation protocols and resource allocation mechanism have been defined that provide proactive behavior to agents for coordination. This architecture has been applied to a real scenario in a collaborative virtual environment for learning. The solution enables users to coordinate with other team members.
\end{abstract}

Keywords: Human interaction with autonomous agents, Cooperation, Dialogue Management, Decision-Making, Resource Sharing

\section{Introduction}

In collaborative virtual environments (VE) for training, human users, namely learners, work together with autonomous agents to perform a collective activity. The educational objective is not only to learn the task, but also to acquire social skills in order to be efficient in the coordination of the activity with other team members 2]. The ability to coordinate with others relies on common grounding [8] and mutual awareness [17. Common grounding leads team members to share a common point of view about their collective goals, plans, and resources they can use to achieve them [8. Mutual awareness means that team members act to get information about others' activities by direct perception or information seeking, and to provide information about theirs' through dialogues [17.

Collaboration in a human-agent teamwork poses many important challenges. First, there exist no global resource that human team members and virtual agents can rely on to share their knowledge. Second, the structure of coordination between human-agent team members is open by nature: virtual agents need to adopt the flexibility of human behavior, as users may not necessarily strictly follow the rules of coordination. In contrast, in agent-agent interactions, agents follow the rigid structure of interaction protocols (e.g., contract net protocol). 
Thus, the ability to coordinate with human team members requires to reason about their shared actions, shared resources and, about the situations where team members need the coordination. Moreover, in human-human teamwork, team members pro-actively provide information needed by other team members based on the anticipation of other's need of information [11].

This paper focuses on the task-oriented collaborative conversational behavior of virtual agents in a mixed human-agent team. Other aspects of spoken interaction with embodied virtual agents, such as non-verbal behaviors, perception, auto speech recognition, and text to speech etc. are out of the scope of this study. As team members must have the shared understanding of skills, goals and intentions of other team members, we proposed a belief-desire-intention based (BDI-like) agent architecture named as collaborative-Conversational BDI agent architecture ( $\left.\mathrm{C}^{2} \mathrm{BDI}\right)$. The contributions of this paper include: (1) definition of collaborative conversational protocols to establish mutual awareness and common grounding among team members; (2) resource allocation mechanism for effective coordination through the means of communication; and (3) decisionmaking mechanism where dialogues and beliefs about other agents are used to guide the action selection mechanism allowing agents to collaborate with their team members. The approach consists in formalizing the conversational behavior of the agent related to the coordination of the activity, which reduces the necessity to explicitly define communicative actions in the action plan.

In section 2, we present related work on human-agent teamwork. Section 3 presents different components of our architecture. The conversational behavior is detailed in section 4 . The next section illustrates how the solution fulfils requirements of real educational scenarios. Finally, section 6 summaries our positioning.

\section{Related Work}

Both AI and dialogue literature agree that to coordinate their activities, agents must have the joint-intention towards achieving the group goal 9 and must have the common plan [12. The joint-intention theory specifies that agents have common intentions towards the group goal [9], whereas the shared-plan theory [12] specifies that even agents share a common plan, it does not guarantee that agents have the commitment towards the group to achieve shared goal. The $\mathrm{C}^{2} \mathrm{BDI}$ architecture takes advantage of these theories to establish common grounding and mutual awareness among human-agent team members.

Numbers of human-agent team models have been proposed. Collagen agent [16] is built upon the human discourse theory and collaborates with a user to solve domain problems such as planning a travel itinerary. In [4], collaboration in teams are governed by teamwork notification policies, where agents inform the user when an important event occurs. To achieve collaboration, [19] proposed a four stage model that includes (i) recognising potential for cooperation, (ii) team formation (iii) plan formation, and (iv) plan execution. Based on this model, [10] describes how collective intentions from the team formation stage are build-up using information-seeking speech act based dialogues. Moreover, 3] proposed an 
agent based dialogue system by providing dialogue acts for collaborative problem solving between a user and a system. In contrast, $\mathrm{C}^{2} \mathrm{BDI}$ agents coordinate with team members not only at the beginning, but also during the realisation of the shared task. Recently, [11/13] have proposed a theoretical framework on proactive information exchange in agent teamwork to establish shared mental model using shared-plan [12]. Furthermore, sharing common resources among team members requires coordination mechanism to manage resource usage. In [15, authors have proposed a negotiation based model, in which agents negotiate to maximise the resource utilisation. In contrast, $\mathrm{C}^{2} \mathrm{BDI}$ agent, based on the anticipation of others' needs, provides opportunities to other members to choose resources.

One of the prominent approaches for dialogue modelling is the information state (IS) approach [18. The IS defined in [5] contains contextual information of dialogue that includes dialogue, semantic, cognitive, perceptual, and social context. This model includes major aspects to control natural language dialogues. However, it does not include contextual information about the shared task. This leads to an incoherence between dialogue context and shared task in progress. In [14, an IS based interaction model for Max agent has been proposed that considers coordination as an implicit characteristic of team members, Comparing with [14, $\mathrm{C}^{2} \mathrm{BDI}$ agents exhibit both reactive and proactive conversational behaviors, and explicitly handle cooperative situations between team members. Moreover, [5] proposed a taxonomy of dialogue acts $(\mathrm{DIT}++)$ based on the dialogue interpretation theory. Semantics of these dialogue acts are based on the IS based approach. We are motivated to use it to understand and interpret conversation between human-agent team due to its following characteristics: (i) it is mainly used for annotation and interpretation of dialogues in human-human conversation; (ii) it supports task oriented conversation; and (iii) it has become the ISO 24617-2 international standard for dialogue interpretation using dialogue acts.

\section{$3 \quad \mathrm{C}^{2} \mathrm{BDI}$ Agent Architecture}

In this section, we describe components of $\mathrm{C}^{2} \mathrm{BDI}$ agent architecture that provide deliberative and conversational behaviours for collaboration (Fig. 1). The agent architecture is based on the theory of shared-plan [12] and joint-intention [9].

The $\mathrm{C}^{2} \mathrm{BDI}$ agent is considered to be situated in an informed VE [6]. It perceives VE through the perception module. The current perceived state of VE is an instantiation of concepts the agent holds in its semantic knowledge. The perception allows agents to enrich their knowledge, and to monitor the progress of the shared activity. Since, the state of VE can be changed due to interactions by team members, the belief revision function periodically updates knowledge base of the agent, and maintains its consistency. It ensures the coherence of the knowledge elements acquired from the perception of the $\mathrm{VE}$ and from the natural language dialogues. The dialogue manager allows an agent to share its knowledge with other team members using natural language communication. It supports both reactive and proactive conversation behavior, and ensures coordination of the activity. The decision-making uses private beliefs and beliefs about others to 


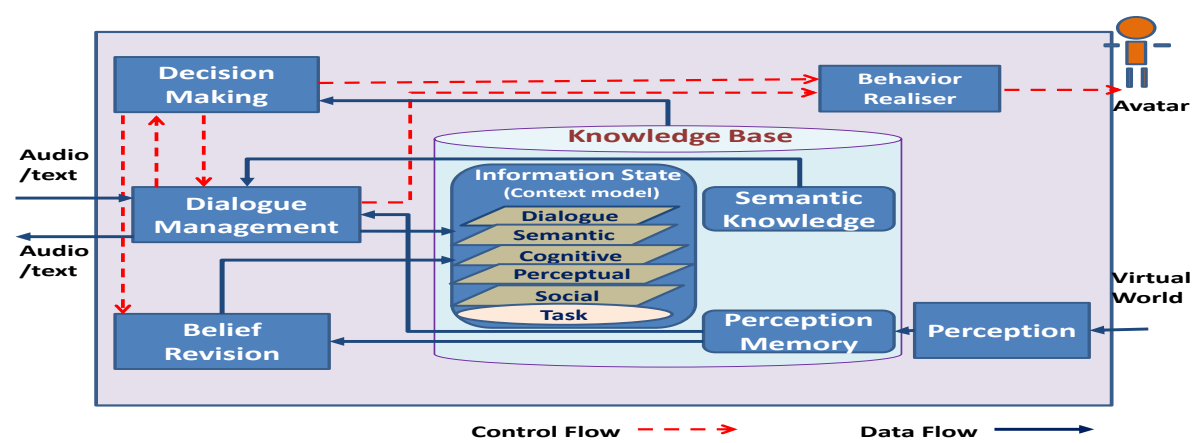

Fig. 1: Components of Agent architecture and data flow

decide whether to elaborate the plan, to identify collaborative situations, to react in the current situation, or to exchange information with other team members. The behavior realiser module is responsible for the execution of actions and the turn taking behavior of the agent.

\subsection{Knowledge Organisation and processing}

The knowledge base consists of semantic knowledge, perception memory and IS (Fig. 1). The semantic modeling of VE [6] is used as semantic knowledge. It contains semantic information that is known a priori by the agent, such as knowledge concerning concepts, and individual and shared plans. Following the shared-plan theory [12], $\mathrm{C}^{2} \mathrm{BDI}$ agents share the same semantic knowledge about VE and the group activity. Sharing the same semantic knowledge supports proactive conversation behavior of the agent, as first, it allows the decision-making process to identify collaborative situations and information needed by other team members, and second, it provides information about the action and resource interdependencies with other team members. The perception memory acquires information about the state of VE perceived by the perception module, whereas, the IS contains contextual information about the current activity and dialogues.

The natural language understanding (NLU) and generation (NLG) of spoken dialogues is based on the rule based approach [1. When the agent receives an utterance, it uses NLU rules to determine the corresponding dialogue act type [5], and the dialogue contents are identified using the semantic knowledge and the contextual information from the IS. The dialogue manager processes these dialogue acts and updates IS based on update rules similar to [18. When the agent has the communicative intention, it constructs dialogue act move and NLG rules are used to generate utterance corresponding to it based on the current context from IS.

\subsection{Information State}

The IS is primarily used in literature [18,5] to control natural language dialogues. The semantic context of the IS is instantiated from concepts the agent holds in semantic knowledge, depending on the progress of the shared task. It includes the 
agenda that contains dialogue goals. To cooperate with other team members, the agent needs not only the information about the current context of the collective activity, but also beliefs about team members to establish common grounding and mutual awareness. To acquire these information, we extend the IS based context model of [5] by adding the task context to it (Fig. 2). We extended its usage as the source of knowledge between decision-making and conversational behavior of the $\mathrm{C}^{2} \mathrm{BDI}$ agent to establish coherence between these two processes. In $\mathrm{C}^{2} \mathrm{BDI}$ agent, the IS works as an active memory.

The task context includes information about the task. It contains intentions in task-focus, goals, and desires of the agent. The $\mathrm{C}^{2} \mathrm{BDI}$ agent follows the theory of joint-intention [9]. It ensures that each team member has common intention towards the team goal. Therefore, the task context also contains cooperativeinformation that includes beliefs about group-goal, group-desire, group-intention, joint-goal, joint-desire, joint-intention, and joint-commitment. We distinguish among individual, group and joint intentions of the agent.

\begin{tabular}{|c|c|c|}
\hline Dialogue Context & \multicolumn{2}{|c|}{ agent-dialogue-acts, addressee-dialogue-acts, dialogue-act-history, next-moves } \\
\hline Semantic Context & \multicolumn{2}{|c|}{ agenda, qud, communication-plan, beliefs, expected-dialogue-acts } \\
\hline Cognitive Context & \multicolumn{2}{|l|}{ mutual-belief } \\
\hline Social Context & \multicolumn{2}{|c|}{ communication-pressure } \\
\hline Perception Context & \multicolumn{2}{|c|}{ object-in-focus, agent-in-focus, third-person-in-focus } \\
\hline \multirow[t]{2}{*}{ Task Context } & cooperative-info & $\begin{array}{l}\text { group-goal, group-desire, group-intention } \\
\text { joint-goal, joint-desire, joint-intention, joint-commitment }\end{array}$ \\
\hline & task & task-focus, goals, desires \\
\hline
\end{tabular}

Fig. 2: Extended Information State of [5] in $\mathrm{C}^{2} \mathrm{BDI}$ architecture

The group-goal indicates that the agent knows that all team members want to achieve the goal at a time or another. Similarly, group-desire and groupintention can be defined analogously. For an agent a group-intention becomes a joint-intention when the agent knows that this intention is shared by other team members. To form a joint-intention, a necessary condition is that the agent must have individual intention to achieve this goal. Similarly, the semantics of jointdesire and joint-goal indicates that all team members have the same group-desire and group-goal respectively, and all team members know it. Thus, these shared mental attitudes towards the group, specify that each member holds beliefs about other team members, and each member mutually believes that every member has the same mental attitude.

The joint-intention only ensures that each member is individually committed to acting. The agent must also ensure the commitment of others to achieve this shared goal. Agents must communicate with other team members to obtain their joint-commitments. The agent has a joint-commitment towards the group, if and only if, each member of the group has the mutual belief about the same groupgoal, the agent has the joint-intention about to achieve that goal, and each agent of the group is individually committed to achieve this goal. Hence, the IS not only contains information about the current context of the dialogue, but also that of the collaborative task, i.e., beliefs about other team members potentially useful for the agent for its decision-making. 


\section{Conversational Behavior}

The conversational behavior allows $\mathrm{C}^{2} \mathrm{BDI}$ agents to share their knowledge with other team members using natural language communication, and ensures the coordination of the team activity. The agent interprets and generates the dialogues based on semantics of dialogue acts proposed in [5]. To achieve coordination among team members, we propose collaborative conversational protocols, and resource allocation mechanism for the agent.

\subsection{Collaborative Conversational Protocols}

As we want the agent to be proactive and cooperative, we define three collaborative conversational protocols (CCP). They ensure the establishment of collaboration among team members to achieve a group-goal, and its end when the current goal is achieved. Every team member participating in a collaborative activity enters in collaboration at the same time, and remains committed towards the group until the activity is finished.

CCP-1: When the agent has a new group-goal to achieve, it communicates with other team members to establish joint-commitment, and to ensure that every team member use the same plan to achieve the group-goal.

When the agent has one or more group-goals to achieve, and if it has no mutual belief about them, it constructs Set-Q(what-team-next-goal) dialogue act addressing it to the group. By addressing this open question, the agent allows both users and other agents to actively participate in the conversation. If the agent receives the choice of the goal from another team member, it adds mutual belief about group-goal and group-intention to its cognitive context, and adds the belief about joint-goal to the task context. It then confirms this choice by sending positive acknowledgement (by constructing Auto-feedback(positive-ack)).

When the agent receives Set-Q(what-team-next-goal) and has no mutual belief about group-goal, i.e., no other team member has already replied to the question, it can decide to reply based on its response time. It chooses one of the available goals based on its own preference rules, and informs sender by constructing Inform(team-next-goal) dialogue act. When the agent receives positive acknowledgement from one of the team members, it modifies its IS by adding mutual belief about group-goal and group-intention, and belief about joint-goal.

If the agent has joint-goal, but not joint-intention to achieve this goal, the agent needs to ensure that every team member will follow the same plan to achieve group-goal. If the agent has more than one plan to achieve this goal, it constructs Choice-Q(which-plan) act and address it to the group, or if the agent has only one plan for the goal, it constructs Check-Q(action-plan) act addressing to the group. When the agent receives a choice of the plan, or the confirmation of the choice of a plan, it adds joint-intention to its task context. It confirms this by sending positive acknowledgement, and constructs the belief about jointcommitment. When the agent receives Choice-Q(which-plan) or Check-Q(actionplan), and has no mutual belief about group-intention, it constructs Inform (planchoice) or Confirm dialogue act respectively to inform about its plan selection. 
When it receives positive acknowledgement from one of the team members, it adds individual- and joint-commitment to achieve the group-goal.

CCP-2: When the agent has performed all its planned actions of the shared activity, but the activity is not yet finished, agent requests other team members to inform him when the activity will be finished.

The agent generates Directive-request(inform-goal-achieved) to ask other members to inform it when the activity will be finished. When the agent receives this dialogue act, it adds communicative goal Inform(goal-achieved) to its agenda.

CCP-3: The agent who finished the last action of the shared activity, informs other team members that the activity is terminated.

The preconditions for CCP-3 are that the agent believes that it has performed the last action of the collaborative activity, and it has the joint-commitment to achieve group-goal. If preconditions are satisfied, it constructs Inform(activityfinished) dialogue act addressing it to the group. When the agent receives the information that the last action of the activity has been finished, and has the belief about joint-commitment in its task context and has a communicative goal Inform (goal-achieved) to achieve, it constructs Inform(goal-achieved) dialogue act to inform other team members that the goal has been achieved. It then adds the belief about the achievement of the goal, and removes the corresponding intention from the task context. When the agent receives the information about goal achievement, it removes the corresponding intention from the task context, and drops the communicative goal Inform(goal-achieved) if it has.

These protocols add expectations of information from other team members which need to be satisfied. In a human-agent team, the user's behavior is uncertain, i.e., user may not necessarily follow these protocols. As the agent updates their beliefs using perception information which can make the expectation to be true from the observation of actions of user perceived by the agent, or from the information provided by other team members.

\subsection{Resource Allocation between Team Members}

Agents must acquire resources necessary to carry out an action. It acquires a resource when needed, and releases it when it is no more required. The resource allocation mechanism for $\mathrm{C}^{2} \mathrm{BDI}$ agent is described in Algo. 1. The resource can be allocated to an agent when the action to be executed contains explicit declaration of the resource, or when the resource is shareable and constraints on the resource (e.g., maximum number of users) are satisfied. The conflict situation arises when the action contains only the declaration of the type of resource, and there exist resource dependency with other agents. If no instance is available, it constructs Directive-request(inform-resource-release) to ask other team members about the availability of the resource. If at least one instance is available, it constructs Set-Q(what-resource-choice) dialogue act to ask the other agent about resource choice. Then, it chooses the available resource, and informs its choice to other team members by creating Inform(resource-choice) dialogue act. Similarly, an agent informs its choice to the sender if it has received 


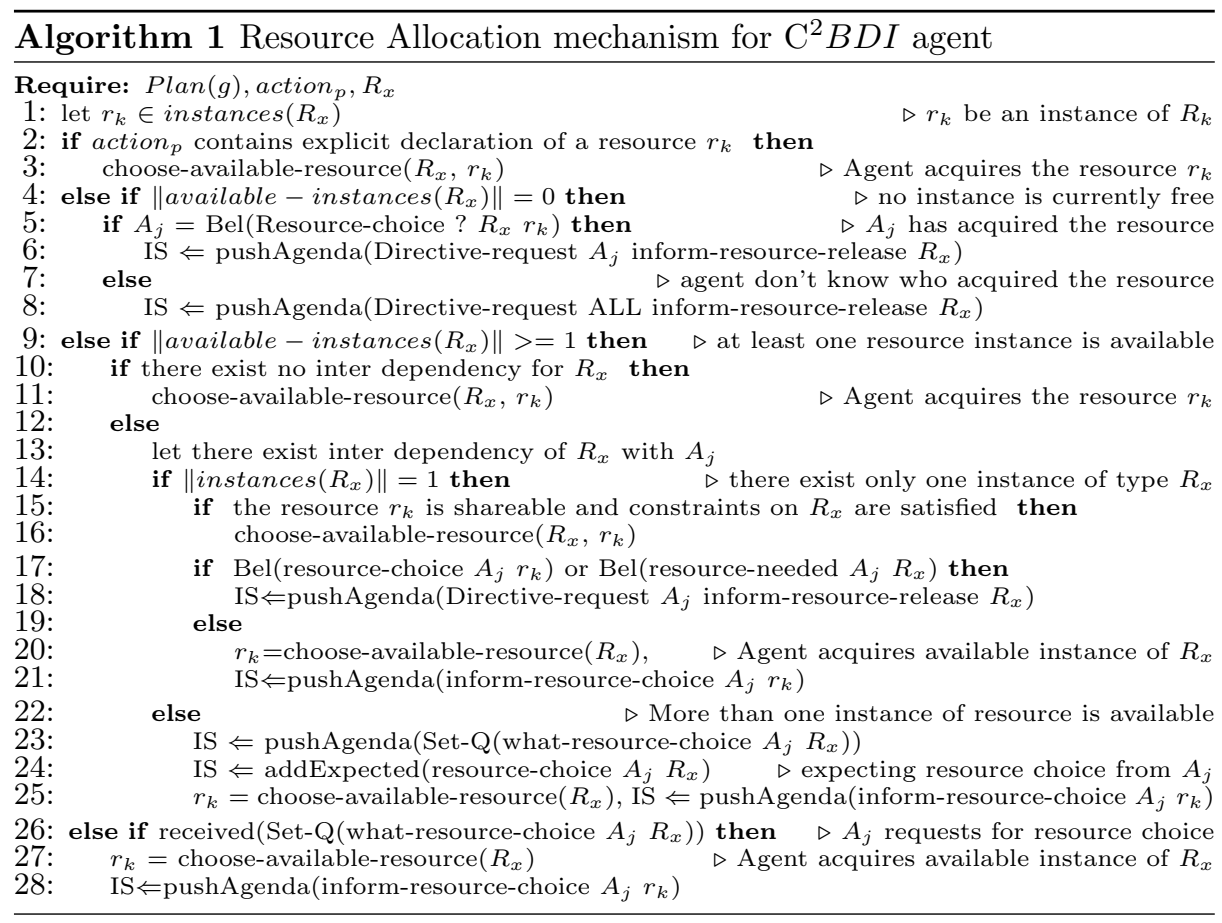

Set-Q(what-resource-choice) request. In this mechanism, agents give chance to other team members to choose resources in the situation of resource conflict.

\subsection{Decision Making}

In $\mathrm{C}^{2} \mathrm{BDI}$ agent, decision-making is governed by information about current goals, shared activity plan, and knowledge of the agent (IS and semantic knowledge). The decision making algorithm is shown in Algo.2. It verifies whether the agenda in IS is not empty or task-focus contains communicative intentions. If so, control is passed to the conversational behavior that supports natural language communication. Otherwise, it chooses the plan to be realised. It identifies cooperative situations in the collective activity where the agent can not progress without assistance. That is, if preconditions for one of the CCPs is satisfied, the control is passed to the conversational behavior. Otherwise, if the agent has an action to be performed that uses a resource, the control is passed to the resource allocation mechanism. These Cooperative situations generate communicative intentions in the agenda that cause the agent to interact with team members to share their knowledge. The agent updates its IS if the control is passed to the conversational behavior, and deliberate the plan to generate a new intention. Once the intention is generated, the agent selects actions to be realised and, updates its task-focus in IS to maintain knowledge about the current context of the task. 


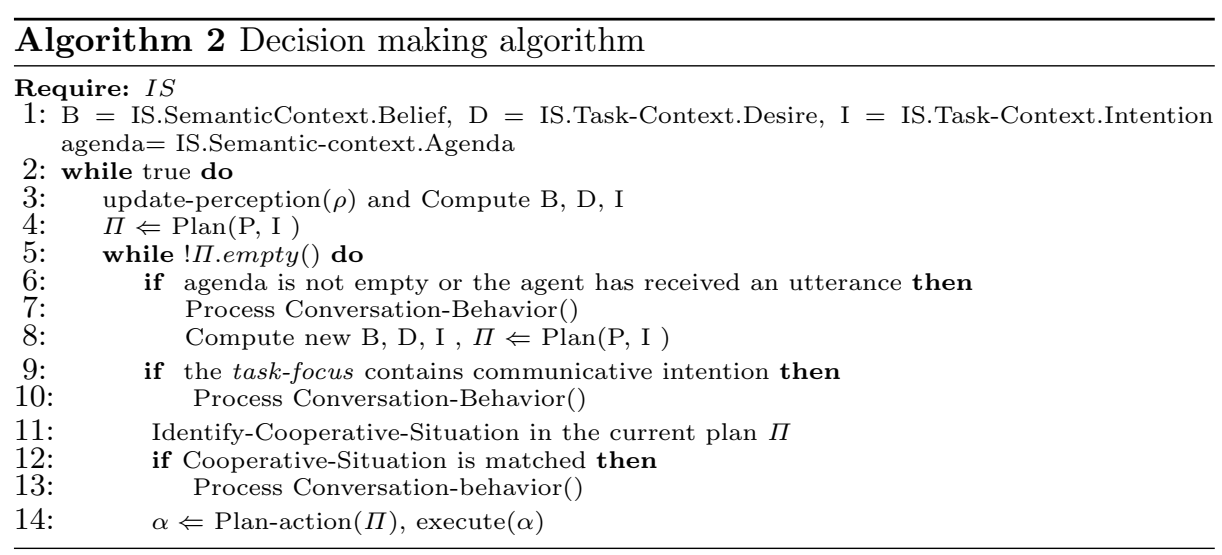

\section{Application Scenario}

Let us consider a motivational scenario where three agents (may include both virtual or real), named as Virginie, Sebastien, and Alexandre need to assemble a furniture. To do so, they need to choose tablets from the table (Fig. 3 left) and place them on shelves (Fig. 3 right). Following sequence of dialogues describe a typical interaction between them where a user plays the role of Alexandre.
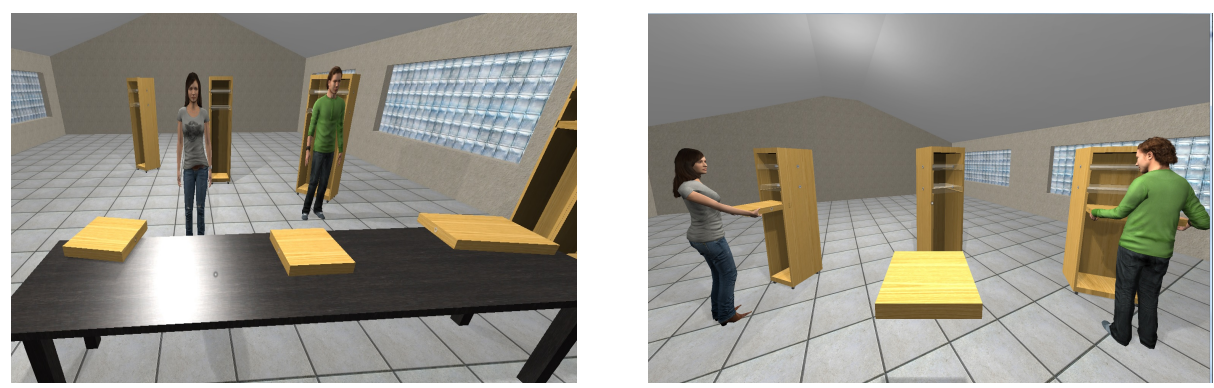

Fig. 3: Furniture Assembly Scenario (First Person view):

left: before tablet selection

right: before choosing tablet position

- S1: Sebastien : What should we do now?

- U1: Alexandre : We should place tablets on shelves.

- S2: Sebastien : $O k$.

- S3: Sebastien : Should we use the place-tablet plan?

- U2: Alexandre: Yes

- S4: Sebastien : I will choose the large tablet.

(Sebastien chooses the tablet near to him and go towards shelf;)

(if user does not make his choice) - V1: Virginie : Alexandre which narrow tablet will you choose? [Set-Q(what-resource-choice)]
- U3: Alexandre : I will choose the left tablet. (user picks the chosen tablet;)

- V2: Virginie : Ok, I will choose the other one.

(Virginie picks the other tablet and go towards the shelf;)

(Sebastien places his tablet on the upper position of the shelf; - S5: Sebastien : Inform me when you will finish the activity. [Directive-request(inform-goalachieved)]

- U4: Alexandre : Virginie which position will you use to place tablet? [Set-Q(what-resourcechoice)]

\footnotetext{
[Inform(resource-choice)]

[Set-Q(team-next-action) [Inform(team-next-goal) [Auto-feedback(positive-ack) [Check-Q(action-plan) Auto-feedback (positive-ack) [Inform(resource-choice)]
} 
- V3: Virginie : I will choose the lower position.

(Virginie places its tablet on the shelf)

[Inform(resource-choice)]

- U5: Alexandre : Ok, I will place my tablet on upper position.

[Inform(resource-choice)]

(User places his tablet on the upper position of the shelf)

[Inform(goal-achieved)]

The challenging scenario includes some important characteristics such as collaborative situations to establish common grounding (S1,U1,S2,S3,U2), handling resource conflicts ( $V 1, U 3, V 2$,), dynamic environment (agents manipulate objects e.g., move tablet), interleaving between communication and actions (agents utter and perform action $\left.S_{4}, U_{3}, V_{3}, U_{4}\right)$, mixed initiative dialogues ( V1,U3, V2 or $U 4, V 3, U 5)$, and both reactive (V3) and proactive $(\mathrm{S} 1, \mathrm{~V} 1)$ communications.

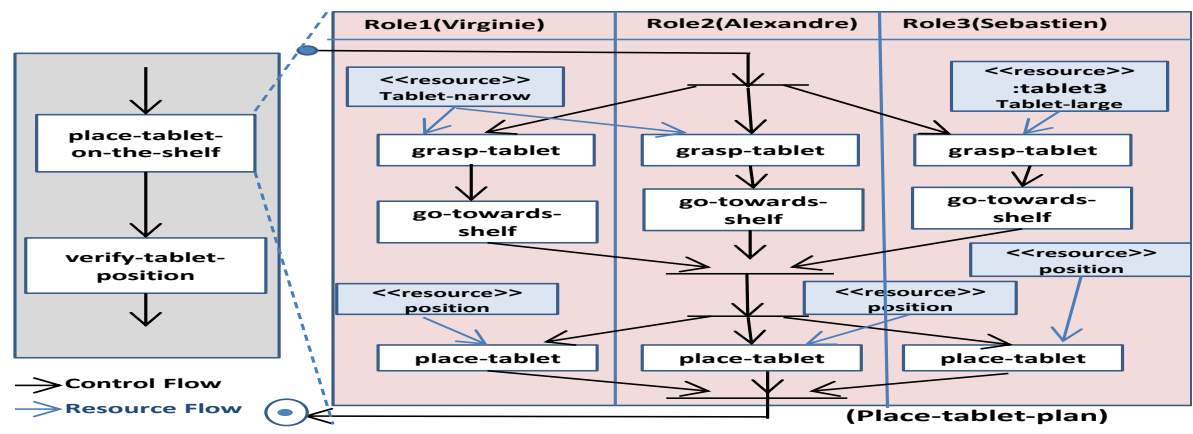

Fig. 4: Partial view of Furniture Assembly plan shared between team members.

Table 1: Snapshot of IS for Virginie and Sabestien before initialisation of CCP-1

\begin{tabular}{|l|l|l|}
\hline & $R_{1}$ (Virginie) & $R_{3}$ (Sebastien) \\
\hline $\begin{array}{l}\text { Information } \\
\text { State }\end{array}$ & $\begin{array}{l}\text { Task-Context(group-goal(" place-tablet-on- } \\
\text { the-shelf") }\end{array}$ & $\begin{array}{l}\text { Task-Context(group-goal("place-tablet-on- } \\
\text { the-shelf")) }\end{array}$ \\
\hline
\end{tabular}

At the beginning, both user and virtual agents have a goal "place-tablet-onthe-shelf". As this goal is shared among team members, it becomes the groupgoal (Fig. 4). A subset of knowledge of agents is shown in Table. 1. Since, Sebastien has a group-goal in its IS, but has no mutual belief about that goal, the decision making process identifies this collaborative situation that fulfils conditions of CCP-1. The CCP-1 generates Set-Q(team-next-goal) dialogue act and generates natural language utterance $S 1$. Sebastien interprets utterance $U 1$

Table 2: Snapshot of IS for agent Sabestien after establishing joint-goal

\begin{tabular}{|l|l|}
\hline & $R_{3}$ (Sebastien) \\
\hline $\begin{array}{l}\text { Information } \\
\text { State }\end{array}$ & $\begin{array}{l}\text { Cognitive-Context(mutual-belief } \\
\text { (group-intention(" place-tablet-on-the-shelf') group-goal(" place-tablet-on-the-shelf')); } \\
\text { Task-Context(group-goal("place-tablet-on-the-shelf") joint-goal(" place-tablet-on-the-shelf') })\end{array}$ \\
\hline
\end{tabular}

as Inform(team-next-goal "place-tablet-on-the-shelf") dialogue act. As Sebastien has the same group-goal, it generates positive acknowledgement S2 for the user and creates mutual-belief about group-goal (Table 2). Virginie passively listens to the conversation and updates its IS following CCP-1. Now, to ensure that the each team member will follow the same action plan, Sebastien construct Check$Q$ (action-plan) dialogue act considering that team members have only one plan 
Table 3: Snapshot of IS of Virginie after establishing joint-commitment

\begin{tabular}{|l|l|}
\hline & Role $R_{1}$ (Virginie) \\
\hline Information & $\begin{array}{l}\text { Cognitive-context(mutual-belief( } \\
\text { group-intention(" place-tablet-on-the-shelf"); group-goal(" place-tablet-on-the-shelf' )); } \\
\text { Task-Context(group-goal("place-tablet-on-the-shelf') joint-goal(" place-tablet-on-the-shelf' }) \\
\text { joint-intention("place-tablet-on-the-shelf' ) joint-commitment("place-tablet-on-the-shelf" }) \\
\text { taskFocus(Intention("grasp-tablet") Intention("place-tablet-on-the-shelf")) })\end{array}$ \\
\hline
\end{tabular}

"place-tablet-plan" to achieve the current group-goal, and generates S3. When both Sebastien and Virginie receive response U2 from user, they construct the joint-intention as well as joint-commitment towards the group-goal and update their IS. The decision making process, now, deliberate the plan and computes the new intention as grasp-tablet (Table 3). Sebastien chooses the large-tablet as the resource is explicitly defined with the action (Algo. 1, line 2). Virginie needs to perform explicit resource acquisition, as only the resource type is defined for its action which is dependent on user's choice (Fig. 4). As two instances of "Tabletnarrow" are available (Fig. 1:left), and if user has not chosen a tablet, Virginie constructs Set- $Q$ (what-resource-choice) to ask user to choose one of the tablets (V1) (Algo. 1, line 22). When user specifies his choice (U3), Virginie chooses the other one (V2). After executing last action "place-tablet" by Sebastien from his plan, and as the shared activity is not yet finished, it utters $S 5$ following CCP-2. When user asks Virginie about its choice of position (U4), Virginie interprets it as Set-Q(what-resource-choice) and informs its choice (V3). Once user places the tablet (U5) which is the last action of the shared plan, Virginie informs all the team members that the goal is achieved (V4) following CCP-3.

\section{Conclusion}

The proposed behavioural architecture $\mathrm{C}^{2} \mathrm{BDI}$ endows agents in the collaborative VE with the ability to coordinate their activities using natural language communication. This capability allows users and agents to share their knowledge. The architecture ensures the knowledge sharing between team members by considering deliberative and the conversation behaviours, not in isolation, but as tightly coupled components, which is a necessary condition for common grounding and mutual awareness to occur. The collaborative conversational protocols and the resource allocation mechanism enable agents to exhibit human-like proactive conversational behavior, that help users to participate in the collaborative activity. While the implemented scenario already shows the benefits of the solution, the behavior of the agents could be enriched both in terms of collaborative team management and in terms of natural language dialogue modelling. Particularly, it would be interesting to endow agents with problem solving capabilities to select their communicative intentions, or to engage themselves into information seeking behaviors and negotiation rounds, as observed in human teamwork [7].

\section{Acknowledgment}

This work was partly supported by the ANR (Corvette project ANR-10-CORD012). 


\section{References}

1. Barange, M., Loor, P.D., Louis, V., Querrec, R., Soler, J., Trinh, T.H., Maisel, E., Chevaillier, P.: Get involved in an interactive virtual tour of brest harbour: Follow the guide and participate. In: Proc. of IVA'11. LNCS, vol. 6895, pp. 93-99. Springer (2011)

2. Barot, C., Lourdeaux, D., Burkhardt, J.M., Amokrane, K., Lenne, D.: V3S: A virtual environment for risk-management training based on human-activity models. Presence 22(1), 1-19 (2013)

3. Blaylock, N., Allen, J.: A collaborative problem-solving model of dialogue. In: Proc. of the SIGdial Workshop on Discourse and Dialog. pp. 200-211 (2005)

4. Bradshaw, J., Feltovich, P., Johnson, M., Bunch, L., Breedy, M., Eskridge, T., Jung, H., Lott, J., Uszok, A.: Coordination in human-agent-robot teamwork. In: Int. Symposium on Collaborative Technologies and Systems. pp. 467-476 (2008)

5. Bunt, H.: The semantics of dialogue acts. In: Proc. of the 9th Int. Conf. on Computational Semantics. pp. 1-13. IWCS '11, Stroudsburg, PA, USA (2011)

6. Chevaillier, P., Trinh, T.H., Barange, M., Devillers, F., Soler, J., De Loor, P., Querrec, R.: Semantic modelling of virtual environments using MASCARET. In: Proc. of the 4th Workshop on Software Engineering and Architectures for Realtime Interactive Systems. Singapore (March 2011)

7. Clancey, W.J.: Simulating activities: relating motives, deliberation, and attentive coordination. Cognitive Systems Research 3, 471-499 (2002)

8. Clark, H.H., Schaefer, E.F.: Contributing to discourse. Cognitive Science 13, 259294 (1989)

9. Cohen, P.R., Levesque, H.J.: Confirmations and joint action. In: Proc. of IJCAI'91. pp. pages 951-957 (1991)

10. Dignum, F., Dunin-Keplicz, Vebrugge, R.: Agent theory for team formation by dialogue. In: Intelligent Agents VII Agent Theories Architectures and Languages. LNCS, Springer Berlin (2001)

11. Fan, X., Yen, J., Volz, R.A.: A theoretical framework on proactive information exchange in agent teamwork. AI 169(1), 23-97 (Nov 2005)

12. Grosz, B.J., Kraus, S.: Collaborative plans for complex group action. AI 86(2), 269 - 357 (1996)

13. Kamali, K., Fan, X., Yen, J.: Towards a theory for multiparty proactive communication in agent teams. Int. J. Cooperative Inf. Syst. 16(2), 271-298 (2007)

14. Kopp, S., Pfeiffer-Lessmann, N.: Functions of speaking and acting: An interaction model for collaborative construction tasks. In: D. Heylen, S. Kopp, S. Marsella, C. Pelachaud et H. Vilhjálmsson, editeurs, The First FML workshop, AAMAS. vol. 8. Portugal (2008)

15. Kraus, S., Wilkenfeld, J., Zlotkin, G.: Multiagent negotiation under time constraints. AI 75(2), $297-345$ (1995)

16. Rich, C., Sidner, C.L., Lesh, N.: Collagen: applying collaborative discourse theory to human-computer interaction. AI Mag. 22(4), 15-25 (Oct 2001)

17. Schmidt, K.: The problem with 'awareness': Introductory remarks on awareness in CSCW. CSCW 11(3), 285-298 (2002)

18. Traum, D., Larsson, S.: The information state approach to dialogue management. In: Kuppevelt, J., Smith, R. (eds.) Current and New Directions in Discourse and Dialogue, Text, Speech and Language Technology, vol. 22, pp. 325-353. Springer Netherlands (2003)

19. Wooldridge, M., Jennings, N.R.: The cooperative problem-solving process. J. of Logic and Computation 9(4), 563-592 (1999) 